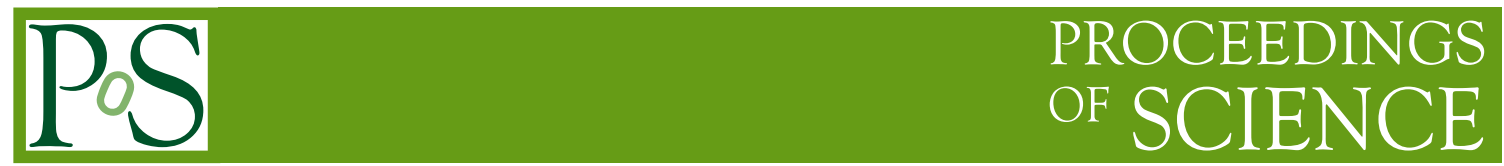

\title{
Multi-leg Hadronic Final State: calculation and simulation
}

\section{Rikkert Frederix*}

Physik Department T31, Technische Universität München, James-Franck-Str. 1, D-85748 Garching, Germany

E-mail: rikkert. frederix@tum.de

In this talk we discuss the recent progess in the computation and simulation of multi-leg hadronic final states. We focus on three recent developments: (i) MINLO as a path NNLOPS, (ii) NLO EW corrections and their matching to parton showers, and (iii) incorporating NLO splitting functions in parton showers.

XXVI International Workshop on Deep-Inelastic Scattering and Related Subjects (DIS2018) 16-20 April 2018

Kobe, Japan

${ }^{*}$ Speaker. 


\section{Introduction}

In the last 10-20 years, there has been a paradigm shift in fully exclusive simulations for collider processes. Essentially moving from the description of data to the prediction of cross sections and differential distributions. The incorporation of physics based on first principles, such as perturbation theory in QCD, allowed for this improved accuracy. While even leading order (LO) multi-jet merging $[1,2,3,4,5,6,7,8,9,10]$ was rarely used for predictions before the start of the LHC, currently many analyses next-to-leading order (NLO) multi-jet merging $[11,12,13,14,15,16,17,18,19,20,21,22]$ has become the new standard. The accuracy of these predictions in unprecedented and allows for a precise scrutiny of all aspects of collider data, with the ultimate goal to either confirm the Standard Model of particle physics also at the energies available at the LHC, or to find physics beyond it.

In this talk, three recent developments will be discussed in more detail: MINLO results for Higgs boson production with up to two jets at NLO accuracy, EW corrections and recent developments towards incorporating NLO splitting functions in parton showers.

\section{MINLO approach as a way to NNLOPS predictions}

Typically, when integrating matrix elements with light partons in the final state over the complete phase space, divergences appear that need to be removed. Most commonly these are removed by requiring a number of jets — defined through an IR-safe jet algorithm - equal to the number of light final state partons in the Born-level contributions to the process, essentially removing the problematic phase space regions. This makes generating accurate and consistent event samples, that can be directly compared to data, independently from the number of jets a formidable task.

One way to solve the above task has been proposed in ref. [21]. This MinLO method allows one to render an NLO calculation for a $B+1$ jet process also NLO accurate for $B$ observables that are completely inclusive over the extra jets (with $B$ a massive colourless system of particles). The general idea is to damp the singularities coming from soft and/or collinear Born-level partons by a suitable Sudakov form factor dampening. By incorporating exactly the right (subset of) higherorder contributions into this form factor, it can be shown that after expanding the results, no terms hamper the NLO accuracy of the observable inclusive in $B$ production.

In the original approach, the precise form of the Sudakov form factor was derived analytically. This allowed for predictions for $W / Z / H / H W / H Z / W W$ production [21, 23, 24, 25]. In all these approaches it was possible to only cover up to one extra jet, i.e. starting from the NLO $B+1$ jet predictions also the $B$ inclusive observables become NLO accurate. With an additional reweighting over the Born variables, these results could even be made NNLO+PS accurate [26, 27, 23, 25, 28].

While the higher order terms in the Sudakov form factor that are needed to get NLO accuracy for the underlying $B$ process are rather simple, this is no longer true for more involved processes. Therefore, in ref. [29] an alternative method was developed. Instead of computing the higher order terms, they are fitted, by imposing that, after integrating over the radiative phase-space, the $B+1$ jet process becomes equal to the $B$ process, in the complete $B$ phase-space. This allows one to determine the required higher order Sudakov terms. In ref. [29] this was applied to Higgs production in gluon fusion. In particular, the start is an NLO calculation for $H+2$ jets, which was made also 
NLO accurate in $H+1$ jet observables. The latter are already part of an NNLO+PS calculation for Higgs production [30]. In short, this allows one to have NLO accuracy for $H+2$ jets and $H+1$ jet and NNLO accuracy for inclusive $H$ observables. ${ }^{1}$

The most interesting result from this exercise is the plot of fig. 1 . In this plot, the transverse momentum of the Higgs boson is plotted, requiring two additional jets in the event, vetoing events with more or fewer jets. In the left panel, three predictions are shown: in red are the new extended MINLO results, in green the central value of the NNLOPS calculation for inclusive $H$ production and in blue the predictions for $H+2$ jets are shown. The latter contains Sudakov resummation, but is formally not NLO accurate for lower multiplicity observables. In the three panels on the right, the ratio w.r.t. the three central values is taken, respectively. The coloured band is the uncertainty coming from scale variations in the respective results. As expected, when the transverse momentum of the Higgs boson is small (compared to the typical transverse momenta of the jets) the extended MiNLO results agree with the calculation for $H+2$ jets. However, when the transverse momentum of the Higgs gets larger, the events are dominated by the Higgs recoiling against a hard jet, that either splits into two, or radiates a softer secondary jet. The latter approach is better described by the NLO predictions for $H+1$ jet: indeed, the extended MINLO results agree with the NNLOPS results for inclusive $H$ production. A much larger set of results and comparisons can be found in Ref. [29].
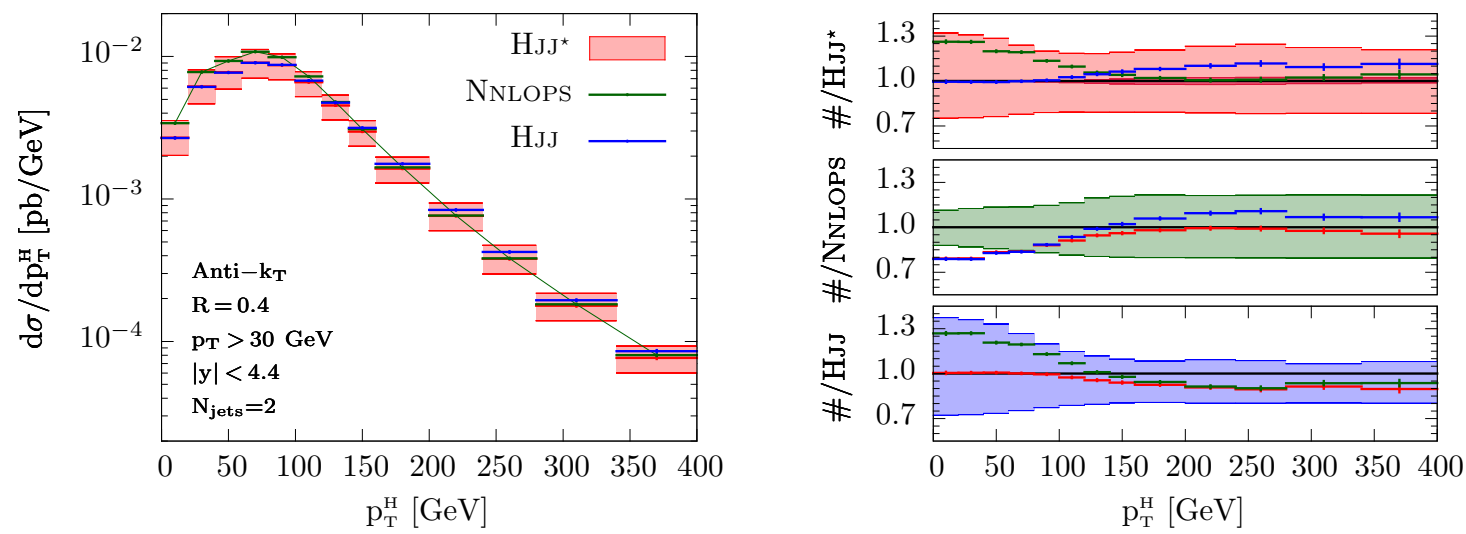

Figure 1: Transverse momentum distribution of the Higgs boson in 2-jet events. Jets are here constructed according to the anti- $k_{t}$ clustering algorithm, for a radius parameter $R=0.4$. Jets are required to have transverse momentum $p_{\mathrm{T}} \geq 30 \mathrm{GeV}$ and rapidity $|\mathrm{y}| \leq 4.4$. Figure taken from ref. [29]

\section{EW corrections}

Naively, by comparing the size of the coupling constants, the EW corrections $\mathscr{O}(\alpha)$ are of the same size as the NNLO QCD corrections $\mathscr{O}\left(\alpha_{s}^{2}\right)$. On top of that, in certain regions of phase space, the EW correction can be much larger than expected from the size of the coupling constant. In particular, close to EW resonances radiation from the decay products results in sizeable effects. Also, with large invariants, EW Sudakov corrections can be large, enhancing the EW corrections

\footnotetext{
${ }^{1}$ Very recently, the same method was also applied to $t$-channel single-top production [31].
} 
to be of similar size, or even larger than NLO QCD corrections. Hence, not only when extreme accuracy is required, but also for tails of distributions, EW corrections are important.

Only for the most simple process, a consistent combination of EW corrections and parton showers is available $[32,33,34]$. On the other hand, recently a lot of progress went in the automation of the computation of EW corrections to any collider process, see e.g. ref. [35]. However, the automation of the matching of NLO EW corrections to parton showers is an unsolved problem, even though some approximated results are on the market, mostly pursued within the Sherpa framework [36, 37, 38].

In particular in ref. [37] the $p p \rightarrow 2 l 2 v$ processes are studied. Both NLO QCD and EW corrections are included. Most interestingly, in a comparison to the exact NLO EW results, two methods of including the resummation of (soft) photons is included. One based on the YFS resummation principle [39], and another on a (dipole) parton shower [40]. In fig. 2 the invariant mass of the 4lepton system is shown, making use of the MC truth to reconstruct the neutrino momenta. As can be seen from the lower left inset, both in the low invariant mass, as well as the large invariant mass regions, the EW corrections are dominating over the QCD ones. In the low invariant mass, this is mostly coming from radiation from the final state leptons, that decrease the 4-lepton invariant mass. A small shift in invariant masses close to resonance peaks can lead to large effects, in particular just below resonance peaks. At large invariant masses, the EW Sudakov logarithms become important, showing $-50 \%$ effects around $m_{l l v v}=5 \mathrm{TeV}$.

The approximated version of the EW corrections do not include hard photon radiation; rather all real-emission contributions are removed, together with the divergent part of the virtual corrections as well as some of the finite terms in the virtual corrections. Of course, this procedure has a great amount of freedom; even so, the results can be compared to the exact results and one finds rather convincing results.

Due to the omission of the photon radiative contributions, when comparing the approximated NLO $Q C D \times E W$ to the exact result (dashed red curve in the lower right ratio plot) the large enhancements close at low invariant mass mostly disappear. However, including the CSS shower (dotted green curve) to reinstate the photon radiation, the predictions overestimate the exact results by significant amounts. This is due to the fact that the recoil of the photon changes the invariant masses of all (pairs of) particles in the process. The YFS method (solid purple curve), however, keeps the pair-invariant masses fixed, and using that to fill-up the photon radiation brings the final predictions much closer to the exact results.

\section{Towards NLO parton showers}

Parton showers are based on LO splitting functions. By exponentiation and re-interpretation them as splitting probabilities, a statistical model for the splitting of partons is constructed. Even though formally only the complete LO information is included, some large NLO terms are considered as well, most importantly due to the choice of renormalisation scale that enters the strong coupling as well as through exact momentum conservation. Recently, some great improvements have been made to include complete NLO splitting functions. In particular, this requires one to move away from the traditional $1 \rightarrow 2$ (or $2 \rightarrow 3$ ) branching functions and include $1 \rightarrow 3$ (or $2 \rightarrow 4$ ) functions as well. 

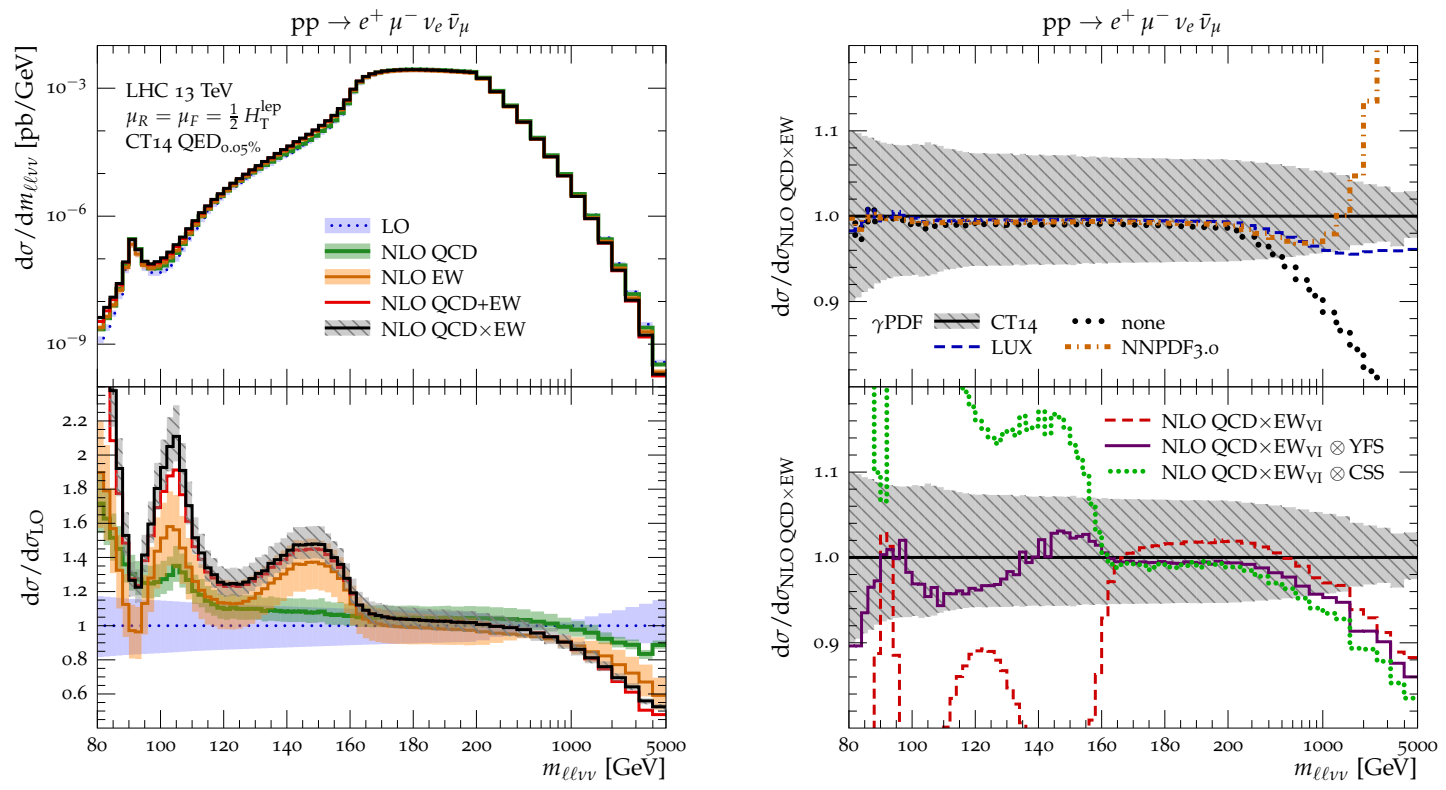

Figure 2: Distribution in the invariant mass of all four final state leptons and neutrinos, $m_{l l v v}$, for $p p \rightarrow$ $e^{+} \mu^{-} v_{e} \bar{v}_{\mu}$ at $13 \mathrm{TeV}$. The left panel shows the absolute predictions and relative corrections with respect to LO. The bands correspond to factor-two scale variations. The upper- right panel shows the effect, at NLO $\mathrm{QCD} \times \mathrm{EW}$ level, of switching off photon-induced contributions or applying photon densities from different current PDFs, while using quark and gluon densities from the nominal CT14qed set throughout. The lowerright ratio shows the level of agreement of the NLO $\mathrm{QCD} \times \mathrm{EW}$ (approx), NLO QCD $\times \mathrm{EW}($ approx $) \otimes$ YFS and NLO $\mathrm{QCD} \times \mathrm{EW}($ approx $) \otimes \mathrm{CSS}$ approximations with the exact NLO QCD $\times \mathrm{EW}$ calculation. Figure taken from ref. [37]

In ref. [41] it was shown that the inclusion of two consecutive $2 \rightarrow 3$ branching together with a direct $2 \rightarrow 4$ can be consistently included in the Vincia program. In particular the inclusion of $2 \rightarrow 4$ branchings opens a phase-space region beyond what can be obtained by strongly ordered $2 \rightarrow 3$ branchings. Moreover, the implementation results in smooth and consistent predictions for differential cross sections, proving that the interplay between $2 \rightarrow 3$ and $2 \rightarrow 4$ branching is understood.

In ref. [42] the complete NLO DGLAP evolution has been implemented within the DIRE parton shower framework, which is available in both Sherpa and Pythia8. However, only in a leading colour and the soft emissions are only LO accuracy. Moreover, given the recent criticism on the DIRE shower [43], the completeness and exactness of the implementation remains an open question, which should be addressed in the near future.

\section{Conclusions}

In this talk, I have focussed on three topics that have received considerable attention in the last year(s).

The first topic is a path to the inclusion of NNLO corrections for processes matched to parton showers. By inclusion of suitable terms in a Sudakov form factor event samples can be simulta- 
neously accurate in $B+1$ jet and $B$ observables. A recent development there is to fit these terms numerically by enforcing the NLO accuracy of observables inclusive over radiation. Having both NLO accuracy for $B+1$ jet and $B$ observables allows one to include NNLO contributions by a simple reweighting in the $B$ phase space to the NNLO predictions for those observables.

The second topic discussed the EW corrections. Recently these have been automated for fixed order predictions. However, when matching to the parton shower, approximations need to be made. First results look promising, but the approximations need to be understood further.

Finally, replacing the LO splitting functions in the parton shower by NLO accurate ones is an ongoing project. Major breakthroughs in the understanding of many of the important issues have recently been made.

In the recent years a lot freedom in tuning the partons showers has been replaced by accurate theory predictions resulting in more predictive power, better control on systematic uncertainties and a greater trust in our measurements. With the availability of the above above improvements in the near future a big step in improving the accuracy of predictions will be taken once again.

\section{Acknowledgements}

The speaker is supported by the Alexander von Humboldt Foundation, in the framework of the Sofja Kovalevskaja Award Project "Event Simulation for the Large Hadron Collider at High Precision".

\section{References}

[1] S. Catani, F. Krauss, R. Kuhn and B. R. Webber, JHEP 0111 (2001) 063 doi:10.1088/1126-6708/2001/11/063 [hep-ph/0109231].

[2] L. Lonnblad, JHEP 0205 (2002) 046 doi:10.1088/1126-6708/2002/05/046 [hep-ph/0112284].

[3] F. Krauss, JHEP 0208 (2002) 015 doi:10.1088/1126-6708/2002/08/015 [hep-ph/0205283].

[4] S. Mrenna and P. Richardson, JHEP 0405 (2004) 040 doi:10.1088/1126-6708/2004/05/040 [hep-ph/0312274].

[5] N. Lavesson and L. Lonnblad, JHEP 0507 (2005) 054 doi:10.1088/1126-6708/2005/07/054 [hep-ph/0503293].

[6] J. Alwall et al., Eur. Phys. J. C 53 (2008) 473 doi:10.1140/epjc/s10052-007-0490-5 [arXiv:0706.2569 [hep-ph]].

[7] S. Hoeche, F. Krauss, S. Schumann and F. Siegert, JHEP 0905 (2009) 053 doi:10.1088/1126-6708/2009/05/053 [arXiv:0903.1219 [hep-ph]].

[8] K. Hamilton, P. Richardson and J. Tully, JHEP 0911 (2009) 038 doi:10.1088/1126-6708/2009/11/038 [arXiv:0905.3072 [hep-ph]].

[9] L. Lonnblad and S. Prestel, JHEP 1203 (2012) 019 doi:10.1007/JHEP03(2012)019 [arXiv:1109.4829 [hep-ph]].

[10] L. Lonnblad and S. Prestel, JHEP 1302 (2013) 094 doi:10.1007/JHEP02(2013)094 [arXiv:1211.4827 [hep-ph]]. 
[11] N. Lavesson and L. Lonnblad, JHEP 0812 (2008) 070 doi:10.1088/1126-6708/2008/12/070 [arXiv:0811.2912 [hep-ph]].

[12] K. Hamilton and P. Nason, JHEP 1006 (2010) 039 doi:10.1007/JHEP06(2010)039 [arXiv:1004.1764 [hep-ph]].

[13] S. Hoche, F. Krauss, M. Schonherr and F. Siegert, JHEP 1108 (2011) 123 doi:10.1007/JHEP08(2011)123 [arXiv:1009.1127 [hep-ph]].

[14] W. T. Giele, D. A. Kosower and P. Z. Skands, Phys. Rev. D 84 (2011) 054003 doi:10.1103/PhysRevD.84.054003 [arXiv:1102.2126 [hep-ph]].

[15] S. Alioli, K. Hamilton and E. Re, JHEP 1109 (2011) 104 doi:10.1007/JHEP09(2011)104 [arXiv:1108.0909 [hep-ph]].

[16] S. Hoeche, F. Krauss, M. Schonherr and F. Siegert, JHEP 1304 (2013) 027 doi:10.1007/JHEP04(2013)027 [arXiv:1207.5030 [hep-ph]].

[17] R. Frederix and S. Frixione, JHEP 1212 (2012) 061 doi:10.1007/JHEP12(2012)061 [arXiv:1209.6215 [hep-ph]].

[18] S. Plätzer, JHEP 1308 (2013) 114 doi:10.1007/JHEP08(2013)114 [arXiv:1211.5467 [hep-ph]].

[19] S. Alioli, C. W. Bauer, C. J. Berggren, A. Hornig, F. J. Tackmann, C. K. Vermilion, J. R. Walsh and S. Zuberi, JHEP 1309 (2013) 120 doi:10.1007/JHEP09(2013)120 [arXiv:1211.7049 [hep-ph]].

[20] L. Lönnblad and S. Prestel, JHEP 1303 (2013) 166 doi:10.1007/JHEP03(2013)166 [arXiv:1211.7278 [hep-ph]].

[21] K. Hamilton, P. Nason, C. Oleari and G. Zanderighi, JHEP 1305 (2013) 082 doi:10.1007/JHEP05(2013)082 [arXiv:1212.4504 [hep-ph]].

[22] S. Alioli, C. W. Bauer, C. Berggren, F. J. Tackmann, J. R. Walsh and S. Zuberi, JHEP 1406 (2014) 089 doi:10.1007/JHEP06(2014)089 [arXiv:1311.0286 [hep-ph]].

[23] W. Astill, W. Bizon, E. Re and G. Zanderighi, JHEP 1606 (2016) 154 doi:10.1007/JHEP06(2016)154 [arXiv:1603.01620 [hep-ph]].

[24] K. Hamilton, T. Melia, P. F. Monni, E. Re and G. Zanderighi, JHEP 1609 (2016) 057 doi:10.1007/JHEP09(2016)057 [arXiv:1606.07062 [hep-ph]].

[25] W. Astill, W. Bizoń, E. Re and G. Zanderighi, [arXiv:1804.08141 [hep-ph]].

[26] K. Hamilton, P. Nason, E. Re and G. Zanderighi, JHEP 1310 (2013) 222 doi:10.1007/JHEP10(2013)222 [arXiv:1309.0017 [hep-ph]].

[27] A. Karlberg, E. Re and G. Zanderighi, JHEP 1409 (2014) 134 doi:10.1007/JHEP09(2014)134 [arXiv:1407.2940 [hep-ph]].

[28] E. Re, M. Wiesemann and G. Zanderighi, arXiv:1805.09857 [hep-ph].

[29] R. Frederix and K. Hamilton, JHEP 1605 (2016) 042 doi:10.1007/JHEP05(2016)042 [arXiv:1512.02663 [hep-ph]].

[30] K. Hamilton, P. Nason, E. Re and G. Zanderighi, JHEP 1310 (2013) 222 doi:10.1007/JHEP10(2013)222 [arXiv:1309.0017 [hep-ph]].

[31] S. Carrazza, R. Frederix, K. Hamilton and G. Zanderighi, arXiv:1805.09855 [hep-ph]. 
[32] L. Barze, G. Montagna, P. Nason, O. Nicrosini and F. Piccinini, JHEP 1204 (2012) 037 doi:10.1007/JHEP04(2012)037 [arXiv:1202.0465 [hep-ph]].

[33] L. Barze, G. Montagna, P. Nason, O. Nicrosini, F. Piccinini and A. Vicini, Eur. Phys. J. C 73 (2013) no.6, 2474 doi:10.1140/epjc/s10052-013-2474-y [arXiv:1302.4606 [hep-ph]].

[34] C. Bernaciak and D. Wackeroth, Phys. Rev. D 85 (2012) 093003 doi:10.1103/PhysRevD.85.093003 [arXiv:1201.4804 [hep-ph]].

[35] R. Frederix, S. Frixione, V. Hirschi, D. Pagani, H.-S. Shao and M. Zaro, arXiv:1804.10017 [hep-ph].

[36] S. Kallweit, J. M. Lindert, P. Maierhofer, S. Pozzorini and M. Schönherr, JHEP 1604 (2016) 021 doi:10.1007/JHEP04(2016)021 [arXiv:1511.08692 [hep-ph]].

[37] S. Kallweit, J. M. Lindert, S. Pozzorini and M. Schönherr, JHEP 1711 (2017) 120 doi:10.1007/JHEP11(2017)120 [arXiv:1705.00598 [hep-ph]].

[38] C. Gütschow, J. M. Lindert and M. Schönherr, Eur. Phys. J. C 78 (2018) no.4, 317 doi:10.1140/epjc/s10052-018-5804-2 [arXiv:1803.00950 [hep-ph]].

[39] D. R. Yennie, S. C. Frautschi and H. Suura, Annals Phys. 13 (1961) 379. doi:10.1016/0003-4916(61)90151-8

[40] S. Schumann and F. Krauss, JHEP 0803 (2008) 038 doi:10.1088/1126-6708/2008/03/038 [arXiv:0709.1027 [hep-ph]].

[41] H. T. Li and P. Skands, Phys. Lett. B 771 (2017) 59 doi:10.1016/j.physletb.2017.05.011 [arXiv:1611.00013 [hep-ph]].

[42] S. Höche, F. Krauss and S. Prestel, JHEP 1710 (2017) 093 doi:10.1007/JHEP10(2017)093 [arXiv:1705.00982 [hep-ph]].

[43] M. Dasgupta, F. A. Dreyer, K. Hamilton, P. F. Monni and G. P. Salam, arXiv:1805.09327 [hep-ph]. 